\title{
Arguments et retour d'expérience sur la distribution d'iode stable autour des centrales nucléaires françaises
}

\author{
B. LE GUEN ${ }^{1}$, P.Y. HÉMIDY ${ }^{2}$, M. GONIN ${ }^{1}$, C. BAILLOEUIL ${ }^{3}$, \\ D. VAN BOXSOM ${ }^{4}$, S. RENIER ${ }^{5}$, Y. GARCIER ${ }^{5}$
}

(Manuscrit reçu le 2 avril 2001, accepté le 7 août 2001)

RÉSUMÉ En cas d'accidents nucléaires, les iodes radioactifs, dont l'iode 131, peuvent être rejetés dans l'atmosphère. Par mesure de sécurité, une instruction du Premier ministre de 1997 ordonne la distribution préventive des comprimés d'iode stable (KI) aux populations voisines des installations nucléaires afin que celle-ci soit déjà effective en situation accidentelle. Auparavant, les comprimés étaient stockés par Électricité de France (EDF) et mis à disposition des pouvoirs publics. Les comprimés distribués en 1997 arrivant à leur date de péremption, EDF a dû mettre en place cette année une nouvelle campagne de distribution dans un rayon de dix kilomètres autour de ses $\mathbf{2 0}$ sites en fonctionnement correspondant à 58 réacteurs nucléaires. Lors des réunions d'information, les questions sur l'efficacité de cette mesure de protection, la nature et la fréquence des éventuels effets secondaires tout en mesurant sa durée d'action selon les conditions d'administration, ont été abordés. Une êtude bibliographique sur la cinétique de l'iode dans l'organisme humain a permis d'établir indications et mode d'emploi. L'efficacité du blocage de l'incorporation d'iode radioactif dans la thyroïde et la survenue d'un dysfonctionnement thyroïdien dépendent de facteurs externes (apports alimentaires d'iode) et individuels (état fonctionnel de la thyroïde, âge du sujet...). Si l'exposition à l'iode radioactif devait durer plusieurs jours, et dans l'objectif d'une protection maximale, l'éventualité d'une deuxième prise d'iode stable pourrait se poser. Ce travail présente l'impact des campagnes d'information (1997 et 2000) et de mise à disposition des populations des comprimés d'iode stable, l'implication des populations, et les réactions des professionnels de santé.

Abstract Arguments and experience feed back for the distribution of iodine tablets in the vicinity of nuclear power plants.

In the event of a nuclear accident, radioactive isotopes of iodine including ${ }^{131}$ I can be released into the atmosphere. In 1997, as a safety measure, the French government decided to begin the distribution of stable iodine tablets (KI) to those living in the vicinity of nuclear power plants, to avoid having to do so in an emergency. The tablets were previously stored by Électricite de France (EDF) which held them at the

\footnotetext{
${ }^{I}$ Électricité de France (EDF), Service central d'appui à la médecine du travail (SCAST), 22-28 rue Joubert, 75009 Paris, France.

${ }^{2}$ EDF, Service de radioprotection, 22-28 rue Joubert, 75009 Paris, France.

${ }^{3}$ EDF, CNPE du Tricastin, Service médecine du travail, BP 9, 26130 Saint-Paul Trois Chateaux, France.

${ }^{4}$ EDF, CNPE du Bugey, Service médecine du travail, BP 14, 01366 Camp de la Valbonne, France.

${ }^{5}$ EDF, Pôle industrie, Division production nucléaire, 1 place Pleyel, 93282 Saint-Denis Cedex, France.
} 
disposal of the government authorities. Since the tablets distributed in 1997 are approaching their use-by date, EDF has started a new distribution campaign within a ten-kilometer radius of its twenty sites with a total of 58 nuclear reactors. During the public information meetings, the discussion focused on the effectiveness of this protective measure and the nature and frequency of the possible side effects while measuring the duration of its action under the conditions in which it was administered. A bibliographic study of the kinetics of iodine in the human body has enabled the indications and the means of use to be determined. The degree of effectiveness with which incorporation of radioactive iodine into the thyroid is prevented and the onset of thyroid dysfunction depend on both external and individual factors: uptake of iodine from food, functional condition of the thyroid, age, etc. In cases of prolonged exposure to radioactive iodine over several days, consideration needs to be given to taking stable iodine a second time, to maintain maximum protection. This presentation covers the impact of the 1997 and 2000 information campaigns, the effect of making stable iodine available to the public, the extent to which the public feels involved, and the reactions of health professionals.

\section{Introduction}

Lors d'un rejet accidentel d'iodes radioactifs dans l'atmosphère, la prise orale d'iode stable par la population a pour objectif de limiter, voire d'empêcher, la fixation thyroïdienne des iodes radioactifs afin de réduire ou d'éviter son irradiation in situ (Crocker, 1984 ; Malarbet et al., 1998 ; Riggs, 1952 ; Saxena et al., 1962).

La glande thyroïde est un des organes les plus radiosensibles de l'organisme (Ramsden et al., 1967), particulièrement chez les personnes jeunes de moins de 18 ans. Suite à l'accident de Tchernobyl en 1986, une augmentation significative de la fréquence des cancers de la thyroïde a été observée chez l'enfant et l'adolescent en Biélorussie, Ukraine et Russie (Jacob et al., 1999). L'exposition aux iodes radioactifs, dont principalement l'iode 131, et l'absence de mise en auvre d'une protection thyroïdienne au moment des rejets, ont joué un rôle prépondérant dans cette augmentation.

Depuis, l'OMS (WHO/CEC, 1990 ; Vulsma et al., 1989), la CIPR (ICRP, 1991) et l'AIEA (IAEA, 1996) ont publié des recommandations pour l'administration d'iode stable en cas d'accident radiologique. En France, une instruction du Premier ministre de 1997 ordonne la distribution préventive des comprimés d'iode stable (KI) aux populations riveraines des installations nucléaires permettant de ne pas intervenir dans l'urgence. Auparavant, les comprimés étaient stockés par Électricité de France (EDF) et mis à disposition des pouvoirs publics.

En 2000, les comprimés distribués en 1997 arrivant à leur date de péremption, EDF a été chargée par les pouvoirs publics de la coordination et de la mise en place 


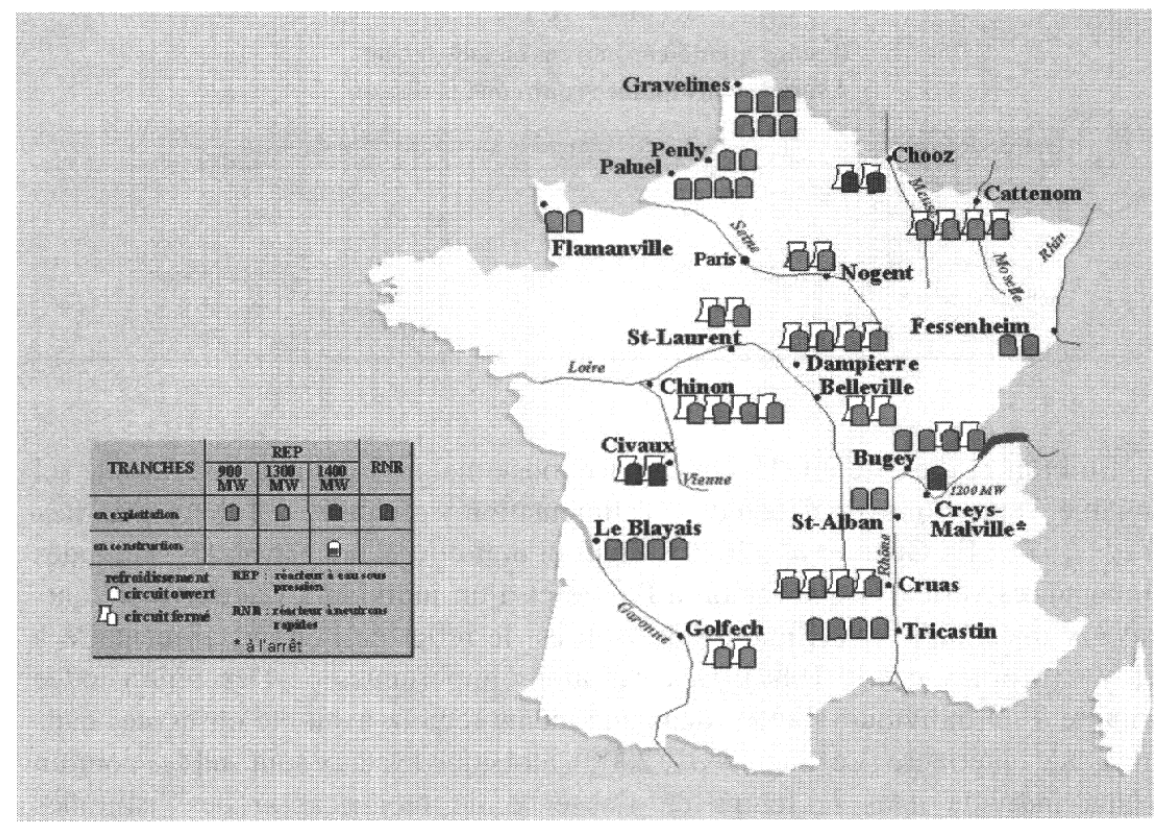

Figure 1 - Localisation des centrales nucléaires EDF en France.

Location of EDF nuclear power plants in France.

d'une nouvelle campagne de distribution dans un rayon de dix kilomètres autour de ses 19 sites nucléaires en activité (Fig. 1). Après avoir rappelé les principales caractéristiques de la chimioprophylaxie iodée en cas d'accident nucléaire majeur, ce travail présente l'impact des deux campagnes d'information et de distribution des comprimés d'iode stable, l'attitude comportementale des populations, et les réactions des professionnels de santé.

\section{Rappels sur le métabolisme de l'iode}

L'iode est un oligo-élément indispensable à la synthèse des hormones thyroïdiennes, la triiodothyronine (T3) et la thyroxine (T4). Il provient essentiellement de l'alimentation et il est admis qu'un apport iodé (Tab. I) de l'ordre de 100 à $150 \mu \mathrm{g} / \mathrm{jour}$ assure la couverture des besoins d'un adulte et de 50 à $100 \mu \mathrm{g} / \mathrm{jour}$ pour un enfant (Pennington, 1990). Pour faire face à une éventuelle carence d'apport, il existe une réserve intra-thyroïdienne de 10 à $15 \mathrm{mg}$ d'iode (Cavalieri et al., 1997). 
TABLEAU I

Besoins quotidiens moyens en iode (en $\mu \mathrm{g})$.

Average daily iodine requirements (in $\mu \mathrm{g}$ ).

\begin{tabular}{|cc|}
\hline Type de persoune & Apports quotidiens nécessaires ( $\mathrm{ug})$ \\
\hline nourrisson & $25-45$ \\
enfant & $50-100$ \\
femme & $100-120$ \\
femme enceinte & $125-200$ \\
homme & 150 \\
\hline
\end{tabular}

En France, il existe une légère carence iodée malgré l'ajout d'iode dans le sel de table, l'apport moyen d'iode dans l'alimentation varie de 85 à $100 \mu \mathrm{g}$ (Mornex et al., 1987 ; Le Guen et al., 2000). Celui-ci doit être nuancé avec les habitudes alimentaires variées d'une région à l'autre et d'un individu à l'autre. Différents travaux ont montré que l'iodurie de $24 \mathrm{~h}$ est le reflet de l'apport alimentaire à $\pm 10 \%$ (Mornex et al., 1987). Des variations importantes de l'excrétion iodée urinaire d'un individu à l'autre, dans une même région, avait été observées dans une étude antérieure (Le Guen et al., 2000), alors que les taux sont stables pour un même individu dans le temps en l'absence de modification des habitudes alimentaires (Fig. 2).

L'iode est absorbé au niveau du tube digestif (estomac, intestin grêle) sous sa forme réduite iodure $\left(\mathrm{I}^{-}\right)$. Une fois dans le flux sanguin, il diffuse rapidement dans le secteur extra-cellulaire, constituant le pool d'iodure extra-cellulaire, et suit deux voies principales, en concurrence :

- le captage par la glande thyroïde et la fabrication des hormones thyroïdiennes, - l'excrétion urinaire (Gaffney et al., 1962).

Le captage de l'iodure par les cellules thyroïdiennes (thyréocytes) est un processus actif. Il s'adapte à la concentration plasmatique d'iodure et donc aux apports alimentaires d'iode. L'ensemble est régulé par une hormone antéhypophysaire, la thyréo-stimuling-hormone (TSH). La thyroglobuline ( $\mathrm{Tg}$ ) constitue une réserve d'hormones correspondant aux besoins de 30 à 60 jours. Leur libération dans la circulation sanguine se fait par protéolyse de la thyroglobuline, également sous l'influence de la TSH. La dégradation enzymatique de la thyroglobuline libère quotidiennement environ $10 \mu \mathrm{g}$ de $\mathrm{T} 3$ et $100 \mu \mathrm{g}$ de $\mathrm{T} 4$ (contenant $70 \mu \mathrm{g}$ d'iode) qui passent dans le plasma. Après action au niveau des tissus cibles, l'iode est libéré et rejoint le secteur extra-cellulaire. Il peut être, soit capté à nouveau par la glande thyroïde (recyclage) et servir à la synthèse hormonale, soit être éliminé pour l'essentiel par le rein. 


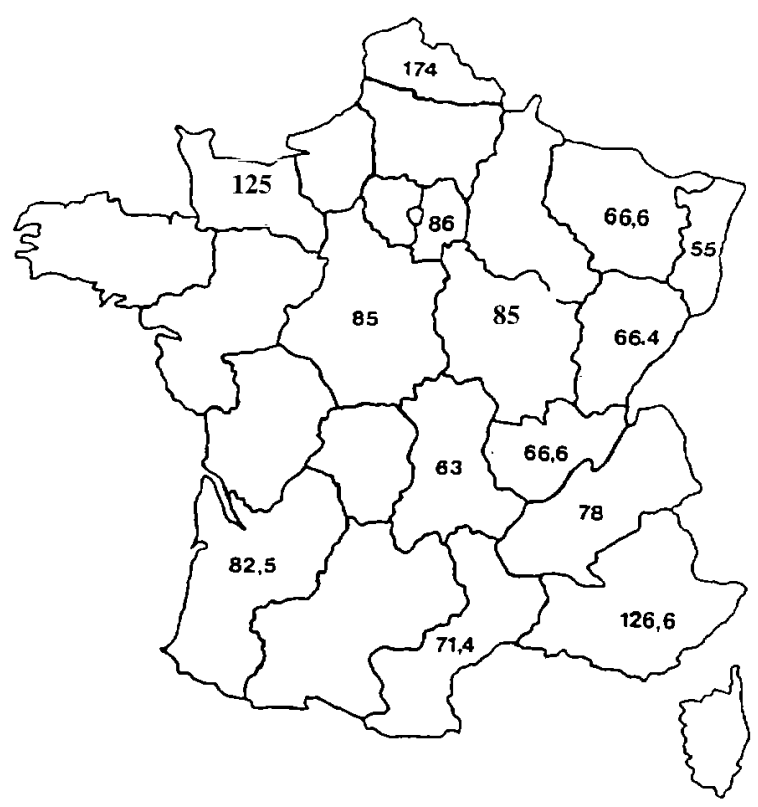

Figure 2 - Iodurie mesurée dans la population de différentes régions françaises.

Comparison of the iodine content in urine in France in $\mu g$ day ${ }^{-1}$ (Mornex, 1987; Le Guen et al., 2000).

Ces rappels permettent de comprendre le mode de fixation des iodes radioactifs sur la glande thyroïde. Ils entrent en compétition avec l'iode stable d'origine alimentaire dont l'apport est insuffisant pour empêcher la fixation des iodes radioactifs (Rubery, 1990). Le risque de cancer chez les enfants et les jeunes adultes, population la plus radiosensible, est donc accru. La prise d'un comprimé d'iode stable avant les rejets d'iodes radioactifs sature la thyroïde en iode et la protège efficacement contre l'absorption des iodes radioactifs (Nauman et Wolff, 1993). Ces derniers sont alors éliminés par voies naturelles. Comme le rappelle le slogan de la campagne 2000 de distribution des comprimés d'iode à la population, « la meilleure protection c'est la prévention ».

\section{Fabrication et organisation de la distribution des comprimés d'iode}

Les comprimés d'iodure de potassium ont obtenu le statut de médicament le 24 janvier 1997 par l'AFSSAPS (Agence française de sécurité sanitaire des produits de santé). Comme tous les médicaments, ils relèvent d'une autorisation 
de mise sur le marché. Cette dernière a été révisée en octobre 1999, sur demande du fabricant, en raison de l'obtention de nouvelles données concernant la stabilité du produit. En effet, l'allongement de la durée de stabilité a été démontré par des études de vieillissement accéléré (variation artificielle de la température ambiante, hygrométrie...) et en temps réel. En conséquence la périodicité de renouvellement des comprimés d'iode a été portée de 3 à 5 ans.

La fabrication de 400000 boites à 6,40 francs de 10 comprimés dosés à $130 \mathrm{mg}$ d'iodure de potassium (KI) (soit $99,38 \mathrm{mg}$ d'élément iode) a été assurée par un établissement pharmaceutique unique, la pharmacie centrale des Armées.

La distribution a suivi la filière pharmaceutique classique :

- livraison des antennes régionales des différents grossistes-répartiteurs,

- acheminement des comprimés d'iode pluri-quotidien aux différentes pharmacies.

Chaque foyer résidant dans un rayon de 10 kilomètres autour d'une centrale EDF a reçu son bon de retrait par courrier, accompagné d'une lettre explicative signée du Préfet. Il est important de préciser que les écoles, les lieux d'activité pour les enfants (centres aérés, crèches), les industries locales et les lieux publics (mairies) ont été également pourvus, en liaison avec les pharmaciens, de stocks de boîtes de comprimés en nombre suffisant.

Les pharmaciens d'officine étant des interlocuteurs privilégiés de santé publique, il a été convenu que les comprimés d'iode seraient retirés dans les pharmacies par la population comme tout autre médicament mais à titre gratuit, sur simple présentation du bon.

La convention signée entre EDF et les représentants de la profession précise par ailleurs que la délivrance devait être accompagnée de conseils sur la prise du médicament (posologie, effets indésirables, contre-indications, conservation...) et de réponses aux interrogations de la population. Pour les aider dans cette tâche, les pharmaciens ont été conviés à de nombreuses réunions d'informations auxquelles ont été associés les services médicaux d'EDF et une documentation abondante leur a été remise.

\section{Thèmes abordés lors des réunions d'information}

\subsection{Posologie et mode d'administration}

Les comprimés d'iode stable ne doivent être utilisés que lors de circonstances exceptionnelles d'exposition et seulement sur ordre des autorités compétentes, en l'occurrence le représentant de l'état de la région concernée (Préfet). 
En France, le niveau d'intervention retenu par les pouvoirs publics est une dose de $100 \mathrm{mSv}$ à la thyroide. Cette valeur se situe dans la fourchette des niveaux recommandés par la publication 63 de la CIPR (ICRP, 1991) et elle est en accord avec les niveaux d'intervention pour les actions urgentes recommandées dans les Safety Series de l'AIEA (IAEA, 1996). Pour les personnes les plus sensibles, âgées de moins de 18 ans, un abaissement du seuil de prise, entre 10 et $100 \mathrm{mSv}$, est discuté (WHO, 1999).

Le traitement consiste en la prise unique d'un comprimé de 130 mg d'iodure de potassium selon les modalités suivantes :

- pour les adultes (hommes + femmes + femmes enceintes) (Evans et al., 1967) et enfants de plus de 12 ans,

- enfants de 3 à 12 ans : la moitié d'un comprimé à dissoudre dans un verre (eau, lait, jus de fruit),

pour les nourrissons de 0 à 3 ans : un quart de comprimé à dissoudre dans un verre (eau, lait, jus de fruit).

\subsection{Efficacité et durée de protection}

Le tableau II fournit l'efficacité de la protection selon le moment de la prise du comprimé d'iode stable pour la prise d'un comprimé d'iode stable de $130 \mathrm{mg}$ d'iodure de potassium. La faible réduction des doses observées lorsque la prise d'iode stable a lieu bien avant l'exposition à l'iode radioactif ( $96 \mathrm{~h}$ ) peut être imputée à l'arrêt de la saturation de la thyroïde. L'efficacité d'une prise unique est de l'ordre de 36 heures. Dans le cas d'une prise tardive, les doses à la thyroïde résultent essentiellement de l'incorporation d'iode radioactif avant l'arrivée d'iode stable.

Le blocage du captage thyroïdien varie en fonction de la posologie d'iodure de potassium mais $90 \%$ de dose évitée à la thyroïde est obtenu dès $20 \mathrm{mg}$ si l'administration d'iode stable a lieu au même moment que l'exposition aux iodes radioactifs (Geoffroy et al., 2000 ; Zanzonico et Becker, 1993).

Du fait de la diminution importante de la protection thyroïdienne au-delà de 36 heures après la première prise d'iode stable (Zanzonico et Becker, 2000) et si l'exposition à l'iode radioactif doit durer plusieurs jours (situation exceptionnelle), l'éventualité d'une deuxième prise d'iode stable, moins réductrice en dose, peut se poser.

Mais cette deuxième prise expose à un risque accru de surcharge iodée. Aussi, afin d'améliorer la tolérance de la chimioprophylaxie mais surtout pour diminuer le risque d'effets indésirables, des auteurs (Koutras et Livadas, 1966 ; 


\section{TABLEAU II}

Efficacité de la chimioprophylaxie iodée pour une inhalation ponctuelle de $1 \mathrm{MBq}$, selon le moment de la prise d'iode stable.

Time tablet is taken/effectiveness of iodine prophylaxis for an intake of $1 \mathrm{MBq}$.

\begin{tabular}{|c|c|c|}
\hline $\begin{array}{l}\text { Délai en heures entre } \\
\text { l'administration d'iodure } \\
\text { de potassium et l'inhalation d'iode } \\
\text { radioactif }\end{array}$ & Dose à la thyroïde en mSv / MBq & Protection thyroïdienne (\%) \\
\hline$-96 \mathrm{~h}$ & 375 & 5 \\
\hline$-72 \mathrm{~h}$ & 267 & 32 \\
\hline$-48 \mathrm{~h}$ & 97 & 75 \\
\hline$-24 h$ & 25 & 93 \\
\hline 0 & 12 & 97 \\
\hline$+2 h$ & 81 & 80 \\
\hline$+8 \mathrm{~h}$ & 235 & 40 \\
\hline$+16 h$ & 329 & 17 \\
\hline$+24 \mathrm{~h}$ & 367 & 7 \\
\hline
\end{tabular}

Ron et al., 1995 ; Verger, 2000) ont publié un schéma thérapeutique optimisé : pour un adulte, un comprimé d'iode stable le premier jour puis un quart de comprimé les jours suivants.

\subsection{Effets secondaires}

Pour la population générale, les effets secondaires sont extrêmement rares et estimés à moins de 3 pour mille $(3,5 \%$ chez l'enfant et $2 \%$ chez l'adulte) (Schlumberger et Pacini, 1997 ; Galles, 1997).

- Ils sont non spécifiques et à type de désagréments (nausées, vomissements, diarrhées, gastralgie, goût métallique dans la bouche) (NCRPM, 1977 ; WHO/CEC, 1990).

- Dans certains cas, on peut observer une hyperthyroïdie (Braverman, 1990 ; Stanbury et al., 1998). Les sujets les plus sensibles sont ceux atteints d'une pathologie thyroïdienne. La plus courante est l'apparition d'une hyperthyroïdie chez les personnes porteuses d'un goitre et âgées de plus de 60 ans (Weaver et al., 1960). Cette complication peut être grave en cas de maladie cardiaque. De rares cas d'hypothyroïdie ont été recensés (Dunn et al., 1998 ; Schober et al., 1976).

- En cas d'allaitement maternel, il faudra veiller au risque de surdosage en iode des nourrissons à qui on aura administré une dose d'iode stable (Castaing et al., 1979 ; Sternthal et al., 1980). Le surdosage, et donc un risque d'effet(s) indésirable(s) accru proviendra du fait que l'iode stable pris par la mère se concentre dans le lait maternel ( $25 \%$ en 24 h) et se surajoute à l'iode administré 
au nouveau-né (Thompson et al., 1994). Par précaution, la question de l'arrêt de l'alimentation au sein pendant 36 heures peut donc se poser.

- Les hypersensibilités avérées à l'iode sont extrêmement rares, de l'ordre de $10^{-7}$ (Nauman et Wolff, 1993). Les réactions allergiques observables suite à l'utilisation de médicament iodé (antiseptique (povidone iodée), produit de contraste) sont essentiellement attribuables au pouvoir immunogène des excipients et non à l'iode (Conn et al., 1996).

L'utilité de la prophylaxie par l'iode stable et son innocuité ont été démontrées en Pologne, où 18 millions de doses ont été distribuées à la suite de l'accident de Tchernobyl. Aucun effet secondaire notable n'a été observé, notamment chez les enfants, les nouveau-nés et les femmes enceintes. Seuls 3 accidents allergiques à type de broncho-spasme ont nécessité une action thérapeutique (Nauman et Wolff, 1993).

\subsection{Contre-indications}

Si le risque d'effet indésirable consécutif à la prise d'iodure de potassium est très faible, il faudra faire attention à quelques rares cas bien identifiés et par définition connus des personnes (NCRPM, 1977 ; Weaver et al., 1960 ; WHO/CEC 1990) :

- hypersensibilité prouvée à l'iode,

- gros goitre avec déviation ou rétrécissement de trachée,

- dermatite herpétiforme,

- pemphigus vulgaire,

- myotonie congénitale.

Les personnes atteintes de ce type de pathologie doivent être informer que des thérapies de substitution peuvent être proposées après un avis médical spécialisé :

- le perchlorate de sodium ou potassium qui entre en compétition avec l'iode,

- les antithyrö̈diens de synthèse (ATS) comme le carbimazole et le propylthiouracile (PTU) qui bloquent l'hormonogénèse thyroïdienne en inhibant l'organification de l'iode.

D’une manière générale, le rapport bénéfice/risque de la prophylaxie à partir de 60 ans est discutable pour certains auteurs car le risque de cancérogenèse radioinduite est quasi-nul mais aussi en raison d'une possible thyrotoxicose induite par l'iode chez les personnes atteintes d'une pathologie thyroïdienne.

En 1999, lors de la proposition de révision des « guidelines » de 1996, l'OMS (WHO, 1999) a proposé l'absence de prévention au-delà de 40 ans et un abaissement du seuil de prise à $10 \mathrm{mSv}$ à la thyroïde pour les personnes âgées de moins de 18 ans, les femmes enceintes ou allaitantes. L'AIEA a été consultée lors de l'élaboration de ce document mais n'a pas souhaité être associée à sa 
publication sans une consultation formelle des états membres et en raison de questions non encore résolues dans ce texte.

En janvier 2001, la FDA (US Food and Drug Administration) a pris position par rapport au document de l'OMS en retenant une prise d'iode stable pour un risque de dose de :

- $50 \mathrm{mSv}$ à la thyroïde chez l'enfant de 0 à 18 ans et pour la femme enceinte ou allaitant,

- $100 \mathrm{mSv}$ pour les personnes de 18 à 40 ans,

- 5 Gy au-delà de 40 ans.

Dans ce dernier cas c'est le risque d'hypothyroïdie qui est prévenu et non le risque de cancer.

\subsection{Alternative iodée au comprimé d'iode}

En cas de situation de crise, les personnes ayant égaré ou n'ayant pu se procurer les comprimés d'iode, peuvent se voir proposer plusieurs alternatives. Elles sont regroupées dans le tableau III. Le risque d'effet indésirable est identique à l'iode sous forme de comprimé, sous réserve du respect de la contre indication que constitue l'iode sous forme d'alcoolé au nourrisson.

\section{TABLEAU III}

Alternatives possibles aux comprimés d'iodure de potassium en cas de situation de crise. Possible alternative to potassium iodine tablets in emergency situations.

\begin{tabular}{|c|c|c|c|}
\hline \multirow[b]{2}{*}{ Présentation } & \multicolumn{3}{|c|}{ Posologies journalières } \\
\hline & $\begin{array}{c}\text { adulte } \\
\text { (y compris les femmes enceintes) }\end{array}$ & $\begin{array}{c}\text { enfants } \\
(1,5 \text { à } 12 \text { ans })\end{array}$ & $\begin{array}{c}\text { noumrissons } \\
\text { ( }<1,5 \text { ans) }\end{array}$ \\
\hline $\begin{array}{l}\text { LUGOL } \\
\text { (solution iodo-iodurée forte) }\end{array}$ & 80 gouttes $=100 \mathrm{mg}$ d'iode & 40 gouttes & 20 gouttes \\
\hline $\begin{array}{l}\text { Teinture d'iode } \\
\text { (solution alcoolique d'iode } \\
\text { officinale) }\end{array}$ & 80 gouttes & 40 gouttes & 20 gouttes \\
\hline Alcool iodé à $1 \%$ & 2 c. à c. & 1 c. à c. & $1 / 2$ c. à c. \\
\hline
\end{tabular}

\section{Bilan des campagnes 1997 et 2000 de distribution des comprimés d'iode}

\subsection{L'information}

Préalablement aux opérations de distribution, des réunions d'information, résultant d'un partenariat entre EDF et les pouvoirs publics, ont été organisées pour les élus, les professionnels de santé (médecins, pharmaciens...) et les 
populations. En parallèle, les médias locaux (presse écrite et radio) ont relayé ces informations. L'implication des élus est à souligner puisqu'elle leur a permis de se rendre acteurs responsables vis-à-vis de la population, ce qui constitue une garantie pour continuer à tisser des liens étroits entre EDF, les élus et la population.

Les réunions de 1997 ont été fortement plébiscitées avec une fréquentation moyenne de 400 personnes par réunion, soit un total d'environ 15000 personnes à travers la France. En revanche, lors du renouvellement de 2000, ce même type de manifestation ne réunissait plus qu'une cinquantaine de personnes par réunion en moyenne. Il est vrai qu'à trois ans d'intervalle, le public concerné avait peu changé.

\subsection{La distribution}

En 1997, différents protocoles de distribution ont été testés :

(1) bon de retrait envoyé chez les résidents,

(2) portage à domicile par les pompiers et/ou la sécurité civile couplé au dépôt d'un bon de retrait en cas d'absence,

(3) envoi postal.

D'emblée, il est intéressant de noter que la distribution à domicile a permis une couverture de la population concernée supérieure à $90 \%$ contre $60-70 \%$ selon les régions pour le retrait dans les pharmacies d'officine.

La campagne 2000 concernait toutes les centrales nucléaires EDF à l'exception du site de Creys-Malville en phase de déconstruction. Les premiers résultats de distribution des comprimés d'iode sont plus faibles avec une moyenne nationale d'environ $43 \%$, malgré les efforts logistiques et financiers importants déployés par EDF. Il a été décidé de ne pas se contenter de ce premier résultat. La majeure partie de la population concernée par cette distribution (> $77 \%$ ) habite dans le rayon des 5-10 km autour du site. Un effort particulier est fait sur cette zone en s'assurant que les écoles et autres lieux accueillant des enfants et/ou du public sont approvisionnés en comprimés d'iode.

Certaines centrales ont donc déjà pris des initiatives pour améliorer le résultat.

- Deux régions ont mis en place une nouvelle campagne d'information des populations. Dans un cas, une lettre du Préfet a été adressée à tous les foyers, remerciant les personnes qui ont retiré leur boite de comprimés et invitant les autres à le faire ; dans l'autre un communiqué de la préfecture est paru dans les journaux locaux. Chacune de ces deux méthodes a entrâné une augmentation de $15 \%$ du taux de distribution. 
- Deux sites ont profité d'un exercice de sécurité civile pour effectuer un rappel sur les comprimés d'iode auprès des populations concernées. Les résultats sont satisfaisants avec près de $70 \%$ de la population couverte. Les exercices semblent être un moyen de relance efficace car ils mobilisent les populations concernées qui sont alors plus réceptives aux messages.

Pour expliquer la diminution de mobilisation de la population locale, plusieurs hypothèses peuvent être émises :

- une accoutumance au risque dans les régions «nucléarisées » depuis de longues années, voire une confiance renouvelée à l'exploitant,

- la population riveraine des CNPE est essentiellement constituée de personnes travaillant ou ayant travaillé sur un site nucléaire,

- l'aspect nouveauté de la première distribution s'est estompé avec le temps,

- l'absence de problèmes dans les centrales a pu contribuer au désintérêt du public pour la campagne de distribution des comprimés d'iode,

- le lancement de la campagne « comprimé d'iode » au début de l'été, juste avant les vacances, n'est peut être pas une bonne formule,

- une diminution de la motivation des professionnels de santé locaux (pharmaciens, médecins...),

- l'implication de tous les acteurs est nécessaire pour réussir à mobiliser les riverains et il est difficile au bout seulement de 3 ans de susciter à nouveau une telle implication.

Outre les réunions à but informatif, la distribution des comprimés d'iode est une occasion pour l'exploitant d'engager un dialogue avec les personnes vivant autour des centrales. Le retrait en pharmacie d'officine fait intervenir beaucoup de paramètres propres à l'implication des personnes (accoutumance au risque, désintérêt...) limitant ainsi la couverture de la population. Les bons résultats ( $>90 \%$ ) obtenus autour de la centrale de Fessenheim en 1997, lors de l'expérience d'un portage à domicile pose la question de l'extension de ce mode de distribution pour la prochaine campagne de 2005.

\section{RÉFÉRENCES}

Braverman L.E. (1990) Iodine induced thyroid disease, Acta Med. Austr. 17, 29-33.

Castaing H. et al. (1979) Thyroïde du nouveau-né et surcharge en iode après la naissance, Arch. Fr. Pédiat. 36, 356-368.

Cavalieri R.R. (1997) Iodine metabolism and thyroid physiology: current concepts, Thyroid. 7, $177-181$.

Conn J.J., Sebastian M.J., Deam D., Tam M., Martin FI. (1996) A prospective study of the effect of nonionic contrast media on thyroid function, Thyroid. 6, 107-110.

Crocker D.G. (1984) Nuclear reactor accidents-the use of KI as a blocking agent against radioiodine uptake in the thyroid-a review, Health Phys. 46, 1265-1279. 
Dunn J.T., Semigran M.J., Delange F. (1998) The prevention and management of iodine-induced hyperthyroidism and its cardiac features, Thvroid. 8, 101-106.

Evans T.C., Kretzschmar R.M., Hodges R.E., Song C.W. (1967) Radioiodine uptake studies of the human fetal thyroid, J. Nucl. Med. 8, I57-165.

Gaffney G.W., Gregerman R.I., Shock N.W. (1962) Relationship of age to the thyroidal accumulation, renal excretion and distribution of radioiodine in euthyroid man, JCEM 22, 784-794.

Galles P. (1997) Toxiques nucléaires, 2nd éd., Masson, Paris.

Geoffroy B., Verger P., Le Guen B. (2000) Pharmacocinétique de l'iode : revue des connaissances utiles en radioprotection accidentelle, Radioprotection 35 (2), 151-174.

IAEA (1996) Intemational basic safety standards for protection against ionizing radiation and for the safety of radiation sources, Safety Series 115, IAEA, Vienna, p. 353.

ICRP (1991) Principles for intervention for the protection of the public in a radiological emergency. Publication 63, Pergamon Press, Oxford, p. 30.

Jacob P., Kenigsberg Y., Zvonova I., Goulko G., Buglova E., Heidenreich W.F., Golovneva A., Bratilova A.A., Drozdovitch V., Kruk J., Pochtennaja G.T., Balonov M., Demidchik E., Paretzke H.G. (1999) Childhood exposure due to the Chernobyl accident and thyroid cancer risk in contaminated areas of Belarus and Russia, Br. J. Cancer 80, 1461-1469.

Koutras D., Livadas D. (1966) The minimum dose of potassium iodide which inhibits the thyroidal radioiodine uptake, Nucl. $\mathrm{Med}$. 5, 256-261.

Kovari M. (1994) Effect of delay time on effectiveness of stable iodine prophylaxis after intake of radioiodine, J. Radiol. Prot. 14, 131-136.

Le Guen B., Exmelin L., Malarbet J.L., Bérard P., Royer P. (2000) Assessment of iodine in the diet of people living around a nuclear reprocessing plant: dose-related consequences of an intake of iodine-129, J. Radioanal. Nucl. Chem. 243 (2), 507-512.

Małarbet J.L., Aurengo A., Roy M., Le Guen B., Devillers C., Métivier H. (1998) Coefficient de dose après incorporation d'iode 129. Influence de l'apport alimentaire, Radioprotection 33, 15-33.

Mornex R. (1987) Enquête sur la prévalence du goitre en France, Bull. Acad. Natl. Méd. 171, 301 -306.

Nauman J., Wolff J. (1993) Iodide prophylaxis in Poland after the Chernobyl reactor accident: benefits and risks, Am. J. Med. 94, 524-532.

NCRP (1977) Protection of the thyroid gland in the event of releases of radioiodine, NCRP Publication 55, National Council on Radiation Protection and measurements, Bethesda, USA.

Pennington J.A. (1990) A review of iodine toxicity reports, J. Am. Diet. Assoc. 90, 1571-1581.

Ramsden D., Passant F.H., Peabody C.O., Speight R.G. (1967) Radioiodine uptakes in the thyroid. Studies of the blocking and subsequent recovery of the gland following the administration of stable iodine, Health Phys. 13, 633-646.

Riggs D.S. (1952) Quantitative aspects of iodine metabolism in man, Pharmacol. Rev. 4, 284-370.

Ron E. et al. (1995) Thyroid exposure to external radiation: a pooled analysis of seven studies, Radiat. Res. 141, 259-277.

Rubery E.D. (1990) Practical aspects of prophylactic stable iodine usage, in lodine prophylaxis following nuclear accidents, Proceedings of a joint WHO CEC Workshop, July 1988, Rubery E.D., Smales E. (Eds.) pp. 141-150, Pergamon Press, Oxford.

Saxena K.M., Chapman E.M., Pryles C.V. (1962) Minimal dosage of iodide required to suppress uptake of iodine-131 by normal thyroid, Science 138, 430-431.

Schlumberger M., Pacini F. (1997) Tumeur de la thyroide, Nucléon, Paris. 


\section{B. LE GUEN et al.}

Schober B., Hunt J.A. (1976) Evaluation of the normal range of values for uptake of radioactive iodine by the thyroid gland, Can. Med. Assoc. J. 115, 29-35.

Stanbury J.B. (1990) The physiological basis for blockade of radioiodine retention by iodine, in lodine prophylaxis following nuclear accidents, Proceedings of a joint WHO/CEC Workshop, July 1988, Rubery E., Smales E. (Eds) pp. 57-64, Pergamon Press, Oxford.

Stanbury J.B. et al. (1998) Iodine-induced hyperthyroidism: occurrence and epidemiology, Thyroid. 8 , 83-100.

Sternthal E., Lipworth L., Stanley B., Abreau C., Fang S.L., Braverman L.E. (1980) Suppression of thyroid radioiodine uptake by various doses of stable iodide, N. Engl. J. Med. 303, 1083-1088.

Thompson J.C. et al. (1944) Cancer incidence in atomic bomb survivors. Part II: Solid tumors. 19581987, Radiat. Res. 137, S-S67.

Vulsma T., Gons M.H., de Vijlder J.J. (1989) Maternal-fetal transfer of thyroxine in congenital hypothyroidism due to a total organification defect or thyroid agenesis, N. Engl. J. Med. 321, 13-16.

Weaver J.C. et al. (1960) Excretion of radioiodine in human milk, J. Am. Med. Ass. 173, 872-875.

WHO/CEC (1990) Report on the joint WHO/CEC Workshop on iodine prophylaxis following nuclear accidents, in Iodine prophylaxis following nuclear accidents, Proceedings of a joint WHO/CEC Workshop, July 1988, Rubery E., Smales E. (Eds) pp. 151-169, Pergamon Press, Oxford.

Wolff J. (1969) Iodide goiter and the pharmacological effects of excess iodide, Am. J. Med. 47, 101-124.

World Health Organization (1999) Guidelines for iodine prophylaxis following nuclear accidents, WHO, SDE/PHE/99.6.

Zanzonico P.B., Becker D.V. (1993) Use of potassium iodide to minimize thyroid radiation from radiactive fall-out, in Iodine deficiency in Europe, F. Delange et al. (Eds) Plenum Press, New York.

Zanzonico P.B., Becker D.V. (2000) Effects of time of administration and dietary iodine levels on potassium iodide (KI) blockade of thyroid irradiation by $131 \mathrm{I}$ from radioactive fallout, Health Phys. 78, 660-667. 